\title{
Requirements for commercial X-ray element-specific imaging technology
}

J C Austin

Submitted 19.11.10

Accepted 31.01.11

\begin{abstract}
Analysis of the composition of material samples nondestructively by means of standard X-ray tube imaging is extremely challenging due to the breadth of the bremsstrahlung spectrum, resulting from mono-energetic electrons striking a thick tungsten target. In previous work, stacks of registered field-flattened images of various samples over an energy range 15-150 keVwere created and analysed. Attempts to remove the effects of the broad spectrum proved the concept of element-specific imaging, but problems still remained. In this work, modification strategies to existing designs are proposed in both hardware and software, which would bolster efforts to remove the effects of the broad X-ray spectrum.
\end{abstract}

Keywords: Element-specific imaging, elemental mapping, greyscale, non-destructive testing, X-ray absorption, $\mathrm{X}$-ray imaging.

\section{Introduction}

Radiography utilising X-rays is a well-established method of imaging the internal structure of opaque objects. Since the beginning of the 20th century, it has become particularly familiar for its contribution to medical diagnosis. Another class of applications has the objective of analysing the chemical or elemental composition of samples. Well known examples include X-ray fluorescence ${ }^{(1,2)}$, magnetic X-ray circular dichroism ${ }^{(3,4)}$ and the use of monochromatic synchrotron sources ${ }^{(5,6)}$ to pick out $\mathrm{K}$-edge discontinuities in X-ray absorption coefficients. However, these methods do not generally present their data in image form. The ability to acquire X-ray images with supplementary compositional data by somehow merging these two classes of technique would be very desirable. In addition, achieving this aim using X-ray tube technology as opposed to monochromatic synchrotron sources would make this hybrid technique significantly more affordable.

Considerable progress has been made in this direction already ${ }^{(7-15)}$. The applications considered relate to forensics, security and authentication of antiquities and artworks. As the technique develops, the applications will widen to include, for example, factory quality control within industry as data acquisition rates are increased. At present, however, the data acquisition process is extremely slow, taking of the order of 30 minutes to complete a single data set. Applications involving live biological samples with medical objectives in mind, for example, are considered too ambitious for the foreseeable future, simply because of the necessity to tightly control and limit X-ray dosage.

Despite this progress, problems still remain. It is the purpose of this paper to highlight the main areas of difficulty with the current method and to suggest improvements in these areas. At this stage it is too early to describe these improvements in detail. Instead, we provide an outline and general strategy on which future

James C Austin is with the University of Keele, Keele, Staffordshire ST5 5BG, UK.Tel: +44 (0)1782 733652; Email: j.c.austin@keele.ac.uk development can be based. The paper is structured in the following way. Section 2 describes the apparatus and summarises the current data acquisition method in terms of an image model. This model describes how the brightness or greyscale of a sample image varies according to the energy setting $\left(\mathrm{kV}_{\mathrm{p}}\right)$ of a maintainable electron gunbased (open) X-ray tube. Section 3 discusses the principal barriers to further improvement. These are the significant breadth of the bremsstrahlung spectrum from a typical X-ray tube and the gross inefficiency of electron/photon conversion at the target. Before concluding, Section 4 deals with strategies for improvements to the X-ray source, the detector and the data/image processing algorithms that comprise the system as a whole. It is demonstrated that no single development can meet all of the requirements but rather a number of smaller incremental improvements over many components of the system.

\section{Current methods}

In previous work $^{(9-15)}$, the apparatus used was a commercial microfocus X-ray imaging system (HMXST225, X-Tek Systems Ltd (Nikon Metrology)). Samples were placed on a five-axis manipulator, which was positioned by stepper motors under computer control. The samples were placed between a 'thick target tungsten anode, open tube' X-ray source and a detector comprising a Tl doped CsI image intensifier (V7739P, Hamamatsu Photonics Ltd), see Figure 1.

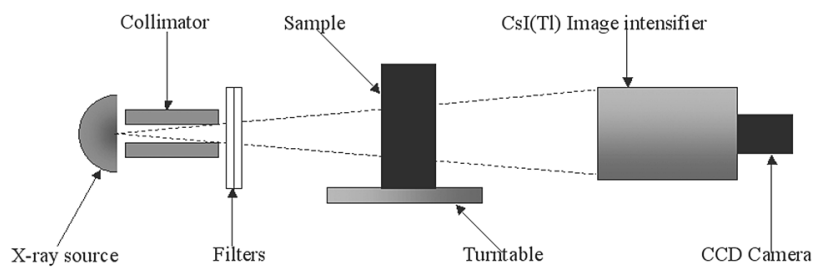

Figure 1. Relative positions of principal microfocus X-ray components

The X-ray source consisted of an electron gun, which was controlled through the system computer. The beam current, incident on the tungsten anode, was controllable over the range $0-1 \mathrm{~mA}$ to a resolution of $1 \mu \mathrm{A}$. The acceleration potential was similarly controllable over the range $15-225 \mathrm{kV}$ in $1 \mathrm{kV}$ steps and was stable to within $\pm 1 / 2 \mathrm{kV}$. The focus spot on the anode was $5 \mu \mathrm{m}$ in diameter and the resulting X-rays were emitted through a beryllium window.

The detector consisted of a $300 \mu \mathrm{m}$-thick Tl-doped CsI diskshaped scintillation screen approximately $100 \mathrm{~mm}$ in diameter as the front face of a photo-multiplier tube. A CCD camera, mounted on the back of the detector assembly, delivered $900 \times 900$ pixel greyscale images with 16-bit greyscale resolution.

The manipulator consisted of a turntable on which the samples were mounted. This had five axes of freedom: $x, y, z$, 'rotate' and 'tilt', with the z-axis (beam axis) corresponding to the line of sight 
between the source and the detector. The (x,y) plane was normal to this, with the $y$-axis vertical. The 'tilt' was a rotational freedom about the horizontal $\mathrm{x}$-axis, whereas the 'rotation' movement was about an axis normal to the surface of the turntable, irrespective of orientation.

In order to optimise conditions for the extraction of spectral features, the X-ray field was shielded by a lead collimator and filtered near to the source. The collimator consisted of a 2 $\mathrm{mm}$-thick lead sheet rolled into a tube approximately $12 \mathrm{~cm}$ long with approximately $8 \mathrm{~mm}$ of wall thickness and an inner diameter of $15 \mathrm{~mm}$. The tube was positioned and aligned along the beam axis. The reason for this was to minimise X-ray scatter from offaxis air and the sides of the cabinet that would otherwise elevate the overall X-ray intensity at the detector, and thereby compromise the signal-to-noise ratio.

Up to three uniform float glass sections $75 \mathrm{~mm}$ square and 6 $\mathrm{mm}$ thick were used as filters. These were orientated in the $(\mathrm{x}, \mathrm{y})$ plane and positioned just beyond the collimator. This had the effect of preferentially attenuating the softer components of the bremsstrahlung flux, hence narrowing the spectrum and shifting its peak towards the more energetic components near to the acceleration potential setting, an effect known as 'beam hardening'.

A typical procedure was to place the sample of interest onto the turntable. With the sample in position, an image, averaged over 256 frames, was taken at a pre-determined lowest energy setting. Under program control the manipulator moved the sample out of shot and a 256 frame averaged 'white' reference image is taken at the same settings. The manipulator motion is then reversed to place the sample in its original position and the acceleration potential is incremented by $1 \mathrm{kV}$. This procedure is repeated until the last pair (sample and 'white' reference) of images is captured at the highest predetermined energy setting. Depending on the sample properties, it was ensured beforehand that the beam current was sufficient for X-rays to penetrate the sample at all potential settings without saturation of the white reference images at any point. The final step was to capture a 'black' reference image, with the detector still on but with the X-ray source off.

The reference images are subsequently used to create a set of aligned (registered) field-flattened images, which have a uniform background. This procedure is sometimes known as background correction or two-point shade correction. The idea is that for any given pixel, the brightness or greyscale of the corrected image, $B$, is given by:

$$
B_{c}=\frac{\left(B-B_{d}\right) W}{B_{l}-B_{d}}
$$

where $B$ is the greyscale of the original image, $B_{d}$ is the greyscale of the black reference (subscript ' $d$ ' for dark), and $B$ is the greyscale for the white reference (subscript ' $l$ ' for light). The factor, $W$, is there to ensure that the corrected image is scaled in the same way as the input images. For images with $n$-bit greyscale resolution we have $W=2^{n}-1$, where in our case $n=16$.

Given a particular sample, it is possible to model the image brightness in terms of its thickness, $d$, and X-ray absorption coefficient, $\mu(E)$, where $E$ is the continuous acceleration potential variable. The sample and white reference greyscales are expressed as functions of acceleration potential, $E_{0}$, ie $B\left(E_{0}\right)$ and $B_{l}\left(E_{0}\right)$, respectively. So the numerator of Equation (1) effectively becomes:

$B\left(E_{0}\right)-B_{d}=\int_{0}^{E_{0}} S\left(E, E_{0}\right) \exp \left(-\mu_{f}(E) d_{f}\right) \exp (-\mu(E) d)\left[1-\exp \left(-\mu_{s}(E) d_{s}\right)\right] d E$

where $S\left(E, E_{0}\right)$ is the spectrum of the X-ray source, which is greater than zero in the range $0<E<E_{0}$ and zero everywhere else. The absorption coefficient and thickness of the filters are given by $\mu_{f}(E)$ and $d_{f}$, respectively, and similarly $\mu_{s}(E)$ and $d_{s}$ are absorption and thickness of the scintillator screen in the detector. The denominator merely consists of a similar expression but with the exponential factor for the sample removed, ie:

$$
B_{l}\left(E_{0}\right)-B_{d}=\int_{0}^{E_{0}} S\left(E, E_{0}\right) \exp \left(-\mu_{f}(E) d_{f}\right)\left[1-\exp \left(-\mu_{s}(E) d_{s}\right)\right] d E \ldots
$$

Equations (1)-(3) represent the basic image model for the greyscale of X-ray images of any sample with known thickness and absorption coefficient. A feature of this technique is that it takes into account the presence of K-edges for elements in the sample being inspected. With known $\mathrm{K}$-edges associated with the filters and scintillation screen it is, in principle, possible to establish the elementary composition of the sample by inferring the spectral positions of K-edges as discontinuities in $\mu(\mathrm{E})$.

\section{The main challenges}

The basic measurement technique and the principles behind it outlined above appear straightforward. However, there are significant practical difficulties to be overcome. The most obvious problem is the broad spectrum associated with X-ray tubes, open or closed, common in many 'off-the-shelf' X-ray systems. The principal aim here is to generate a narrower spectrum with sufficient power flux to maintain good signal-to-noise conditions over a full range of applicable acceleration potentials.

$\mathrm{X}$-ray systems being considered here are a form of shadowgraph, where the X-ray flux must penetrate the sample in order to be detected. If we are examining solid or liquid samples then X-rays with energies less than $\sim 10 \mathrm{keV}$ cannot be considered simply because their wavelength is longer than typical interatomic spacing. This sets a lower limit for K-edge detection. The element with the lowest $\mathrm{K}$-edge above $10 \mathrm{keV}$ is gallium with atomic number 31 . Unfortunately, this immediately excludes analysis of many organic samples, unless we are testing for the presence of heavier target materials such as impurities, for example. Ignoring $\mathrm{K}$-edges for the moment, to a good approximation the X-ray absorption coefficient of an element satisfies an expression of the form:

$$
\mu(E)=\alpha Z^{m} E^{-n}+\beta(E)
$$

where $Z$ is the element's atomic number, $\alpha$ is a constant and the electron scattering term, $\beta(E)$, has a weak dependence on $E$ and so can also be approximated as a constant. The first term on the righthand side, which is atomic number dependent, is due to fluorescence and, unless we are operating at extreme energies $(\sim 10 \mathrm{MeV})$, will dominate the right-hand side of Equation (4). If we approximate the K-edge energy using Moseley's empirical formula, $E_{K} \approx 10.2 Z^{2}$, then the absorption coefficient, ignoring the scattering term, of an element at its K-edge is approximated by:

$$
\mu\left(E_{K}\right) \approx(10.2)^{-n} \alpha Z^{m-2 n}
$$

The powers, $m$ and $n$, are approximated by 2.28 and 2.72 , respectively. These figures are calculated from data published by Hubbell and Seltzer ${ }^{(16)}$, whereas an approximation by Liebhafsky et $a l^{(17)}$ gives 4 and 3 , respectively. Either way the power of $Z$ is negative in Equation (5), implying that for any given effective sample thickness, shade corrected images will tend to be darker for low atomic number elements at the K-edge.

At the other end of the scale the heavy elements tend to have greater physical density, implying a high electron density overall. This results in a high absorption coefficient. Even for uranium the fluorescence term on the right-hand side of Equation (4) still dominates the absorption coefficient at the energy of the K-edge, so the corresponding X-ray image will tend to be dark, albeit for different reasons. If we ignore the transuranic elements and consider uranium to be our heaviest element, then we would need to be able to generate high $\mathrm{X}$-ray flux over an energy range exceeding $10-116 \mathrm{kV}$ (the uranium K-edge is at $115.606 \mathrm{kV}^{(16)}$ ), sufficient to penetrate any sample with a K-edge in that range. Moreover, in these circumstances it would be necessary to plot energies well beyond 
the $116 \mathrm{kV}$ point in order to capture the full effect of the uranium $\mathrm{K}$-edge. Combining this with filtration to narrow the spectrum sufficiently to resolve K-edge discontinuities just compounds the problem further by weakening the $\mathrm{X}$-ray flux.

One of the principal reasons for weak X-ray intensity before we apply filtering is the inefficiency of electron/X-ray conversion at the anode. The electron/photon conversion efficiency, $\eta$, is, using our notation, given by ${ }^{(17)}$ :

$$
\eta=1.4 \times 10^{-9} Z E_{0}
$$

For a tungsten $(Z=74)$ anode this is approximately $1 \%$ per $100 \mathrm{kV}$. So our X-ray system, which is typical, will deliver a maximum of $1 \mathrm{~W}$ of X-ray flux (maximum current $=1 \mathrm{~mA}$ ) at $100 \mathrm{kV}$. There is little to be gained by using heavier elements for the anode target. At $100 \mathrm{kV}$, for instance, using depleted uranium $(Z=92)$ for the target would only increase the efficiency to $1.228 \%$. So this, coupled with limited access to such a target material, more or less closes the door on strengthening the X-ray flux by this method.

An alternative approach might be to use thin target systems where the bulk of the electrons in the beam penetrates the target. This has the advantage of having a narrow spectrum already because electrons interacting with the target are most likely to lose all of their energy. Whereas in their 'thick target' cousins, just described, most of the soft bremsstrahlung tail results from electrons interacting with the bulk of the target, which does not exist for thin target systems. The main problem with this, however, is that most of the electron beam does not interact with the target, so the X-ray intensity would be extremely low to start with.

\section{Towards a dedicated system}

Developing systems dedicated to K-edge-based element-specific $\mathrm{X}$-ray imaging is likely to require incremental changes to a large number of specific design features rather than a single major development. Such a programme will require a redesign of the X-ray source and the detector. Apart from that, the further development of specialist software for the processing of raw images is also needed. On the software side, previous work ${ }^{(9-15)}$ represents the first steps in this direction. So, in summary, there are three areas to concentrate on: (i) the source; (ii) the detector; and (iii) image processing.

\section{The source}

When considering a traditional 'thick target' source using tungsten for the anode material, it would not be necessary for the maximum acceleration potential to exceed around $150 \mathrm{kV}$. Such a potential would exceed the uranium K-edge by a margin sufficient to see its effects without placing too high a demand on the source power supply. At the other extreme $(Z \leq 40)$, a high current is demanded to offset not only the reduced potential, but also the reduced conversion efficiency at lower settings. Therefore, in order to maintain a good X-ray flux at low acceleration potentials, the input power would need to be correspondingly higher. This in turn would put heavy demands on any power supply and cooling system, which would have to be designed accordingly. Most likely the cooling fluid would need to be actively chilled, and a larger target may be required to conduct the extra waste heat away from the beam impact point. The increased beam current would also demand higher field strengths from the focusing coils in order to control the increased charge content of the beam. This would further add to the load on the system.

Another desirable feature would be a source power supply for which the output voltage can be increased in smaller steps or, better still, to be continuously controllable. The latter would enable data capture routines with variable steps in the acceleration potential. In this way, the settings could be clustered close to energies corresponding to the K-edges of desired target elements, and made sparser elsewhere in the spectrum where there is less interest.
Such a supply could be stabilised, for example, by applying feedback through a precision resistor potential divider network on the output. These network resistors must be in close enough proximity for exposure to similar thermal conditions, while at the same time care should be taken to ensure that their proximity does not result in high-voltage breakdown. Submerging the feedback network in high dielectric strength oil, which is presently used on our HMXST225 machine, would most likely be sufficient. The output from the potential divider would supply a signal for comparison with a 'set point' signal with readouts from both. The set point value could, in turn, be controlled by a modified version of the algorithm summarised above. Also, an appropriate test point with readout or waveform display would allow an assessment of the power supply stability. An output stable to within a margin of $\pm 100 \mathrm{~V}$ would be very desirable, although at present the development of such a system would not be without its difficulties given likely budget constraints.

\section{The detector}

As already mentioned, our current detector assembly consists of an X-ray image intensifier (XRII) with a CCD camera mounted at its output. The XRII has more than sufficient sensitivity for the proposed application and has a well-established track record in other similar fields ${ }^{(18-20)}$.

However, there appears to be a significant noise problem with CCD cameras in general. Image noise, associated with CCD camera technology, falls into two broad categories: random noise and fixed-pattern noise. The former, which is due mainly to ambient thermal conditions and affects a whole range of electronic devices, can be eliminated in a non-real-time system by applying more intense X-rays, longer exposures or averaging over more frames. Fixed-pattern noise, on the other hand, is due to a fixed distribution of 'hot pixels' resulting from a variation of element characteristics on the CCD chip.

The reason we limit our averaging to 256 frames is because, in our previous results ${ }^{(9-15)}$, random noise exceeds fixed-pattern noise by an amplitude ratio of approximately $16(=\sqrt{256})$. Therefore, further averaging makes little difference to the overall image noise. A significant improvement, therefore, would be to remove the remaining fixed-pattern noise, which would permit further noise reduction by frame averaging. In principle, fixed-pattern noise should be easy to remove because it is repeatable. However, in order to do this, it would be required to determine how it behaves with variation of image greyscale. For example, if it were additive then subtraction of a dark reference image would be sufficient to remove it. Whereas if its 'hot pixel' intensities were proportional to the greyscale, it would be removed automatically by a two-point shade correction routine like the one we use at present.

The fact that fixed-pattern noise is not eliminated so easily would suggest that 'hot pixel' intensity is not a linear function of the image brightness local to the pixel of interest. One possibility would be to examine intensity deviations from the average over the visual field for a set of field-flattened blank images with increasing brightness. The data obtained could be stored in a 'look-up' table and used to compute a deviation from average brightness at every point in the image, based on a local brightness average. Of course, this data is camera specific, so the exercise would need to be repeated should the camera be changed. The local brightness average in a sample image could be acquired as an average intensity of a pixel along with its eight immediate neighbours. Subtracting the stored deviation at each pixel would reduce the fixed-pattern noise in the sample image significantly. Comparing an image average with a local average of just nine pixels is not ideal and there may arise situations that render it unsatisfactory depending on requirements. In such circumstances it may be necessary to resort to more sophisticated techniques based on those of, for example, Lyu et $\mathrm{l}^{(21)}$ which were used for the removal of fixed-pattern noise 
in spectral data obtained from the Hubbell space telescope.

The use of a flat-panel detector (FPD) as an alternative to the $\mathrm{XRII} / \mathrm{CCD}$ camera combination is another possibility. However, like CCD cameras, FPDs are not free from fixed-pattern noise either ${ }^{(22)}$. In such a case, fixed-pattern noise reduction algorithms would still be required.

Assuming that we could significantly reduce fixed-pattern noise, then this would open the way to further noise reduction by frame averaging. Depending on the condition of the raw data increasing the signal-to-noise ratio in this way would reduce the data acquisition rate. At present, it seems that the only way to mitigate this outcome would be, as already discussed, to increase the X-ray power flux from the source.

\section{Image processing}

The term 'image processing' is normally used in the context of applying filtering techniques to highlight specific features of interest within an image or to improve clarity generally. This is because images are normally regarded as two-dimensional objects. Whereas our data sets are three-dimensional, consisting of stacks of identical images with the third axis representing X-ray energy in the form of the potential setting, $E_{0}$. Given any pixel within the image field, brightness or greyscale can be plotted as a function of X-ray energy. It is this greyscale function/spectrum that is of principal interest. As the X-ray energy setting increases, the broad source spectrum may pass through the K-edge of a sample element imaged at a given pixel location. In so doing, the greyscale function will deviate away from the course expectation based on the K-edge not being present, see Figure 2. The problem is that this deviation is smooth and it is difficult to see where, at the K-edge, it actually starts. This smoothness is due to the breadth of the spectrum, which would not be a problem for near-monochromatic X-rays. Indeed, in that case, the K-edge would show up as a sharp discontinuity in the greyscale spectrum. The challenge here is to find an effective way to highlight the K-edge position in the spectrum, hence identifying the element responsible.

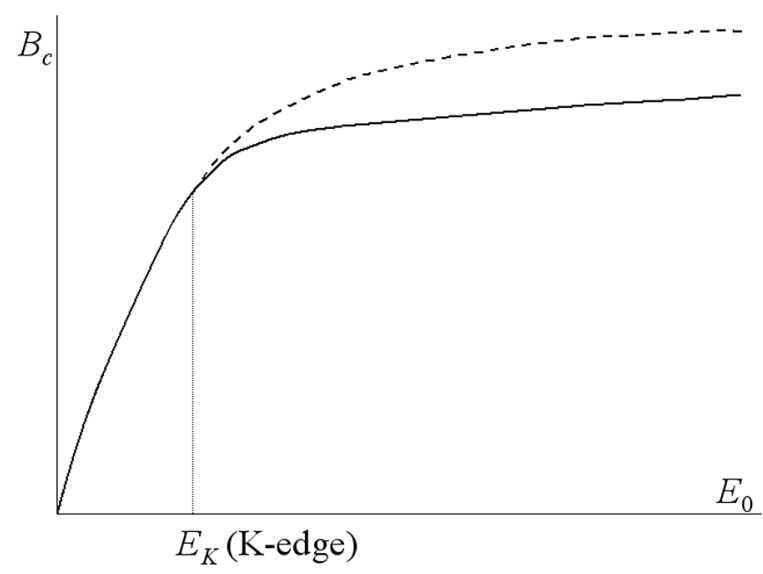

Figure 2. Typical greyscale function for a single element sample with K-edge at $E_{K}$ (solid line). Notional equivalent sample greyscale function with no K-edges in the range covered (dotted line)

Previously we have used the regularisation technique developed by Tikhonov ${ }^{(23,24)}$ to reduce the effect of the spectrum breadth on the greyscale function. While there are problems with this technique, it still remains useful. One particular problem is that it acts as a highpass filter on the greyscale spectrum, which in turn amplifies any 'high-frequency' noise that may be present. Previous suggestions for image noise reduction may go some way to resolving this issue.

Another important point is the fact that the source spectrum is not exactly known. For all of our previous work we used an approximation of the thick-target spectrum based on Liebhafsky et $a l^{(17)}$ given by:

$$
S\left(E, E_{0}\right)=\frac{(p+1)(p+2) E^{p}\left(E_{0}-E\right)}{E_{0}^{p+2}}
$$

where all symbols have their usual meanings and $p$ is a constant in the range 1-3. This spectrum, being an idealised model, is itself a smooth curve, whereas the actual spectrum is likely to be closer to that shown in Figure 3. This is based on an actual spectrum (Amptek Incorporated) from a tungsten thick target source with the acceleration potential set to $100 \mathrm{kV}_{p}$.

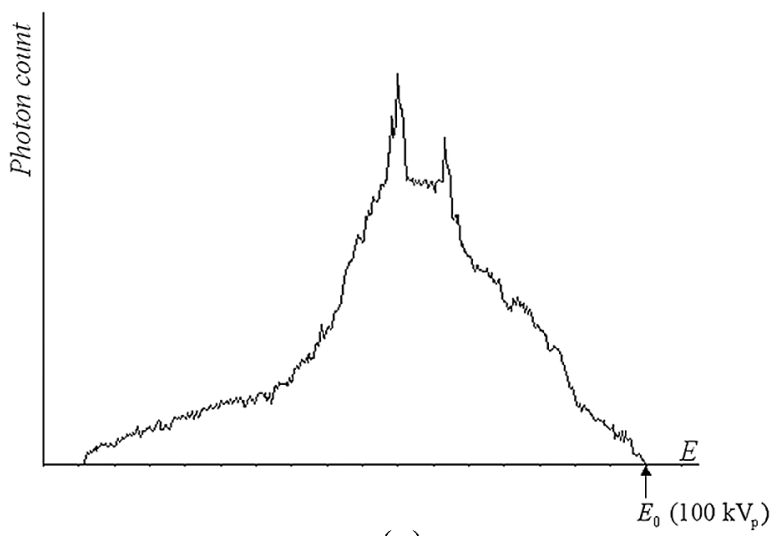

(a)

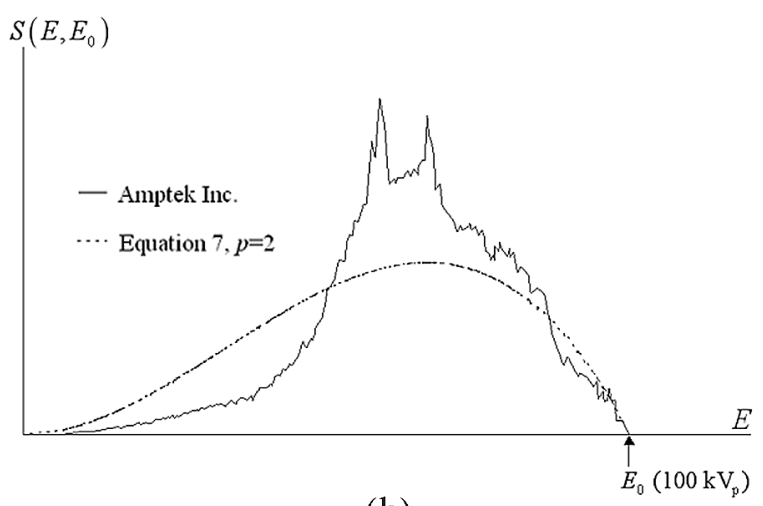

(b)

Figure 3. (a) Typical X-ray spectrum from a thick-target tungsten source at a setting of $100 \mathrm{kV}$ plotted in terms of photon count on the vertical axis (Amptek Incorporated); (b) The same spectrum re-plotted in terms of power intensity (solid line) in comparison with a model spectrum based on Equation (7) with $p=2$ (dotted line)

Because actual thick-target spectra are far from smooth, it is likely that approximating them using the smooth spectrum represented by Equation (7) will introduce rapidly changing components into the output from the Tikhonov process. Briefly, the technique is as follows: substitute Equations (2) and (3) into Equation (1) and express this in the condensed form:

$$
B_{c}\left(E_{0}\right)=\int_{0}^{E_{0}} S\left(E, E_{0}\right) A(E) d E
$$

This is then discretised and written in matrix form $\mathbf{B}_{\mathbf{c}}=\mathbf{S A}$, such that when $S\left(E, E_{0}\right) \rightarrow \delta\left(E-E_{0}\right)$ then $\mathbf{S} \rightarrow \mathbf{I}$ (the identity matrix). Generally $\mathbf{S}$ is a lower triangular matrix with all zeros on the leading diagonal. This has the consequence that the inverse, $\mathbf{S}^{-1}$, does not exist and therefore neither Equation (8) nor its discretised version can be inverted. An approximate inverse is therefore obtained from:

$$
\overline{\mathbf{S}}^{-1}=\left(\mathbf{S}^{\mathbf{T}} \mathbf{S}+h^{2} \mathbf{I}\right)^{-1} \mathbf{S}^{\mathbf{T}}
$$

where $\overline{\mathbf{S}} \rightarrow \mathbf{S}$ as $h \rightarrow 0$, and $h$ is the regularisation parameter. Setting $h$ too close to zero has the effect of amplifying rapidly 
changing components within any of the spectra involved.

As previously mentioned, removing noise from the image data would be beneficial. Further improvement would be gained by using the actual source spectrum in this process rather than that represented by Equation (7). The source spectrum from our microfocus X-ray system would need to be measured over the proposed operating range (10-150 keV) using an $\mathrm{HP}(\mathrm{Ge}) \mathrm{X}$-ray/ gamma-ray spectrometer, of a type supplied by Bruker-Baltic for example. The data would be held in a look-up table and used for the Tikhonov regularisation process. It may be necessary to renew this data periodically to take into account subtle spectral changes that may occur over long periods. How often this would need to be done is yet to be established. The improvements so far suggested represent a likely next stage in the development of broad-spectrum element-specific X-ray imaging.

\section{Conclusion}

An improvement strategy for commercial X-ray imaging systems has been proposed, which would enhance performance for the application of element-specific imaging or element mapping within an X-ray image. There are three principal suggestions that can be incorporated into the design of modern X-ray imaging systems with this aim in mind. These are: (i) the introduction of a facility to measure and store a 'thick-target' spectrum, and associated data processing algorithms for the application of Tikhonov regularisation; (ii) the installation of a similar facility to measure and store 'hot pixel' distribution patterns in the detector for the reduction of fixed-pattern noise; and (iii) the introduction of a precision feedback network on the HV power supply coupled with its re-design to improve stability and enable near continuous control of the output.

Additions to these main design features would include the application of actively-chilled cooling fluid for the anode, coupled with the increased output current from the HV power supply. This would necessitate increasing the current-carrying capacity of the focusing coils to maintain the small microfocus spot size at the anode. These final measures are open-ended and the extent to which they could be taken would be determined by future research and development. Although some of these features may exist on some systems already, for example actively-chilled cooling (X-Tek Systems Ltd (Nikon Metrology)), as far as is known there exists no $\mathrm{X}$-ray system incorporating all of the above design features.

\section{Acknowledgement}

The author gratefully acknowledges Professor Peter W Haycock for his planning and development of the original project (EPSRC, $\mathrm{EP} / \mathrm{C} 008138 / 1)$ on which this work is based.

\section{References}

1. A Wrobel, E Rokita and M Karwala-Szytula, 'Effects of lowfrequency magnetic field on hard and soft tissues elemental compositions', Trac Elem and Elect, 24 (3), pp 125-132, 2007.

2. A Tumuklu, M G Yalcin and M Sonmez, 'Detection of heavy metal concentrations in soil caused by Nigde city garbage dump', Pol J Environ Stud, 16 (4), pp 651-658, 2007.

3. A Ney, T Kammermeier, E Manuel, V Ney, S Dhar, K H Ploog, F Wilhelm and A Rogalev, 'Element-specific investigations of structural and magnetic properties of Gd:GaN', Appl Phys Lett, 90 (25), 2007.

4. A F Bartelt, A Comin, J Feng, J R Nasiatka, T Eimuller, B Ludescher, G Schutz, H A Padmore, A T Young and A Scholl, 'Element-specific spin and orbital momentum dynamics of $\mathrm{Fe} /$ Gd multilayers', Appl Phys Lett, 90 (16), 2007.
5. B Gilbert, G Margaritondo, D Mercanti, P Casalbore and G De Stasio, 'Synchrotron spectromicroscopy in medicine and biology', J Alloys Compd, 328 (1-2), pp 8-13, 2001.

6. A Iida, T Noma, S Hayakawa, M Takahashi and Y Gohshi, 'High-spatial-resolution XAFS and its imaging applications', Jpn J Appl Phys Part 1, 32 (Suppl 32-2), pp 160-164, 1993.

7. J Raistrick, 'X-ray imaging in its element', Mater World, 9 (1), pp 11-13, 2001

8. J C Austin, K E Pitt, P W Haycock, B R Heywood, S D George and J Liddle, 'Broad spectrum element-specific X-ray imaging', NDT\&E Int, 37 (3), pp 239-236, 2004.

9. J C Austin, C R Day, A T Kearon, S Valussi and P W Haycock, 'Using polychromatic X-radiography to examine realistic imitation firearms', Forensic Sci Int, 181 (1-3), pp 26-31, 2008.

10. J C Austin, C R Day, A T Kearon, S Valussi and P W Haycock, 'Characterisation of metallic powder impregnated pastes using polychromatic X-radiography', Insight, 50 (10), pp 550-553, 2008.

11. C R Day, J C Austin, J B Butcher, P W Haycock and A $\mathrm{T}$ Kearon, 'Element-specific determination of X-ray transmission signatures using neural networks', NDT\&E Int, 42 (5), pp 446-451, 2009.

12. J C Austin, C R Day, A T Kearon and P W Haycock, 'Single element mapping in radiography', X-ray Spectrom, 38 (6), pp 492-504, 2009.

13. J C Austin, C R Day, A T Kearon, D L Evans and P W Haycock, 'Comparison method to differentiate painted objects using polychromatic X-rays', Insight, 52 (3), pp 140-143, 2010.

14. K P Lam and J C Austin, 'Visualising multidimensional signals from a dimension-dependent computational perspective', Proc IEEE Intl Conf Signal Image Processing, 2006.

15. K P Lam, J C Austin and C R Day, 'A course-grained spectral signature generator', Proc SPIE, 6356, 63560S, 2007.

16. J H Hubbell and S M Seltzer, 'Tables of X-ray mass attenuation coefficients and mass energy-absorption coefficients from $1 \mathrm{keV}$ to $20 \mathrm{MeV}$ for elements 1 to 92 and 48 additional substances of dosimetric interest', NISTIR 3562, NIST, 1996.

17. H A Liebhafsky, H G Pfeiffer, E H Winslow and P D Zemany, 'X-ray absorption and emission in analytic chemistry', Wiley, New York, 1960.

18. P M Jenneson, W B Gilboy, E J Morton and P J Gregory, 'An $\mathrm{X}$-ray micro-tomography system optimised for low-dose study of living organisms, App Rad and Isotop, 58 (2), pp 177-181, 2003.

19. I Lima, J T de Assis and R T Lopes, 'Three-dimensional conic beam X-ray microtomography in bone quality', Spectrochim Acta Part B Atom Spec, 64 (11-12), pp 11731179, 2009.

20. Y C Li, Y T Wang, S Y Tang and Y Liu, 'Distortion correction and geometric calibration for X-ray angiography system', IEEE Trans Nucl Sci, 56 (3), pp 608-619, 2009.

21. C H Lyu, F C Bruhweiler and A M Smith, 'Tomography power spectrum techniques for removal of fixed pattern noise from Hubble Space Telescope spectra', The Astrophysical Journal, 447, pp 880-888, 1995.

22. C E Floyd, R J Warp, J T Dobbins, H G Chotas, A H Baydush, $\mathrm{R}$ Vargas-Voracek and C E Ravin, 'Imaging characteristics of an amorphous silicon flat-plate detector for digital chest radiography', Radiology, 218 (3), pp 683-688, 2001.

23. A N Tikhonov, 'On the stability of inverse problems', Dokl Akad Nauk SSSR, 39 (5), pp 195-198, 1943.

24. A N Tikhonov, 'Solution of incorrectly formulated problems and the regularisation method', Soviet Math Dokl, 4, pp 1035$1038,1963$. 